\title{
Design of Nanoparticles functionalized with Cell Penetrating Peptides for the treatment of Alzheimer's disease
}

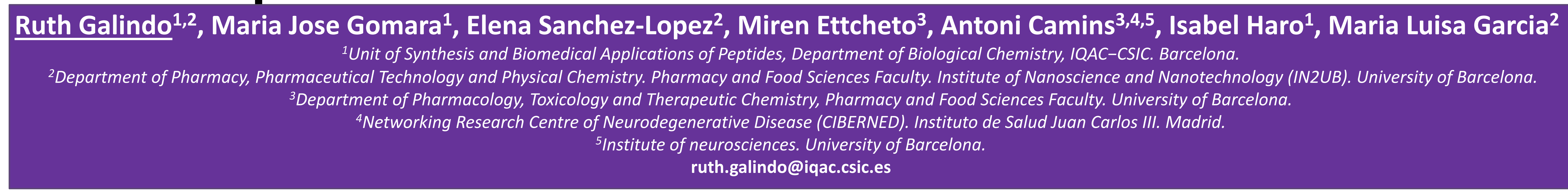

\section{INTRODUCTION}

Neurodegenerative diseases are caused by the progressive death of neurons in different regions of the nervous system. Among these, Alzheimer's disease (AD) is caused by progressive formation of senile plaques and neurofibrillary tangles in the cerebral cortex, as well as the loss of neurons and synapses. The risk of suffering a neurodegenerative disease increases with age, this situation creates a critical need to improve develop new approaches for its treatment. The $\mathrm{N}$ terminal c-Jun kinases present different isoforms JNK1, JNK2 and JNK3, which are involved in the loss of neuronal cells in neurodegenerative diseases. Licochalcone-A (Lic-A), a novel flavonoid, has an inhibitory effect on the activity of JNK1 and could inhibit JNK3. In this way, it could become an innovative alternative to neurodegenerative treatment. However, the presence of the blood-brain barrier (BBB), due to its high selectivity, becomes a limiting factor for drugs to reach the brain tissues.

Nanoparticles (NPs) are colloidal systems of particular interest for drug transport increasing accumulation at the target site and achieving controlled release of the drug. The functionalization of NPs with cell penetrating peptides (CPPs) has attracted attention, because it increases their internalization efficiency in the cell membrane, improving their pass across barriers.

The aim of this work is to develop Lic-A loaded poly(lactic-co-glycolic acid) (PLGA) NPs functionalized with CPPs, suitable to cross BBB and target them to the brain.

\section{METHODS}

Lic-A NPs were prepared by solvent displacement method ${ }^{(1)}$ (Fig. 1) using the polymer PLGA and optimized by central composed factorial design. Morphometrical properties (average size, and polydispersity index, $\mathrm{PI}$ ) of the NPs were determined by photon correlation spectroscopy (PCS) by using a Zetasizer Nano ZS at $25^{\circ} \mathrm{C}$. NPs surface charge, measured as zeta potential (ZP) was evaluated by laser-Doppler electrophoresis with M3PALS system in the same instrument. Morphological studies were carried out by transmission electron microscopy (TEM). Encapsulation efficiency (EE) of drug was determined by HPLC, previous sample ultracentrifugation.

Peptides (TET-1-Cys and B6) were synthesized by solid phase peptide synthesis as C-terminal carboxamides on a NovaSyn TGR resin and following a 9-fluorenylmetoxicarbonyl (Fmoc) strategy ${ }^{(2)}$. The peptides were characterized by analytical HPLC and Electrospray Ionization Mass Spectrometry (ESI-MS) (Fig. 2).
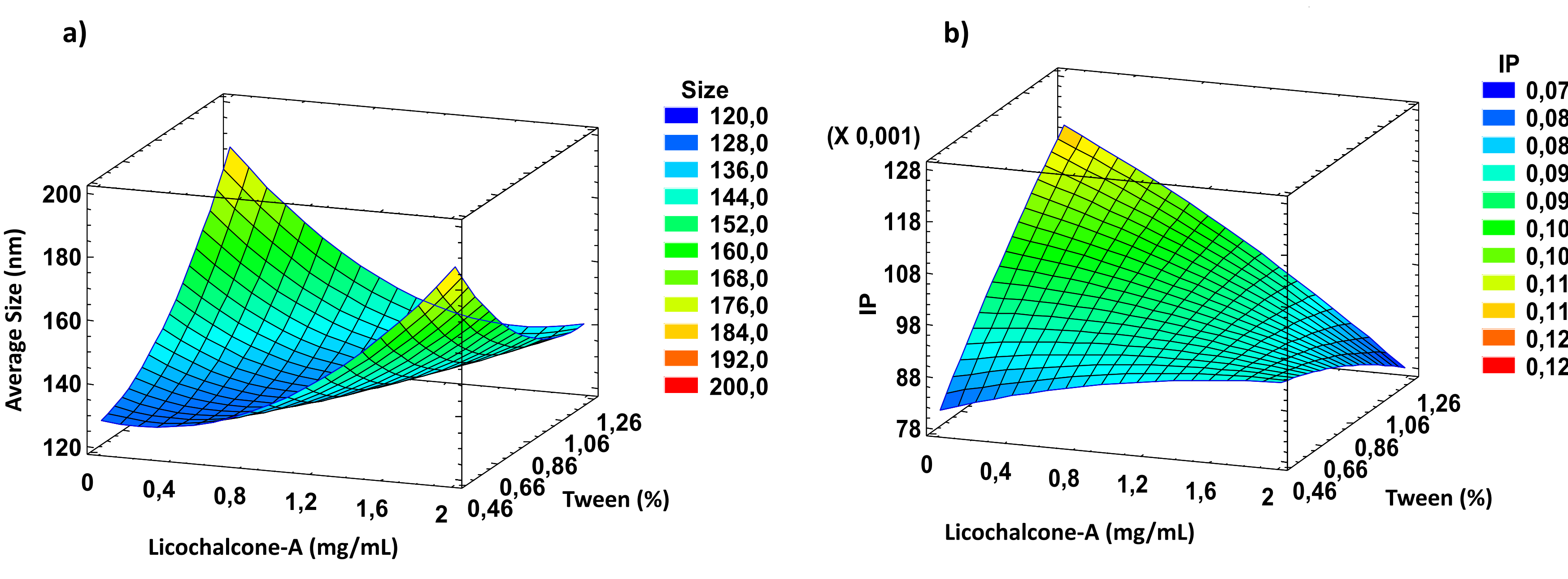

Fig. 3.Response surface plot for Lic-A loaded NPs : a) Average size; b) PI.
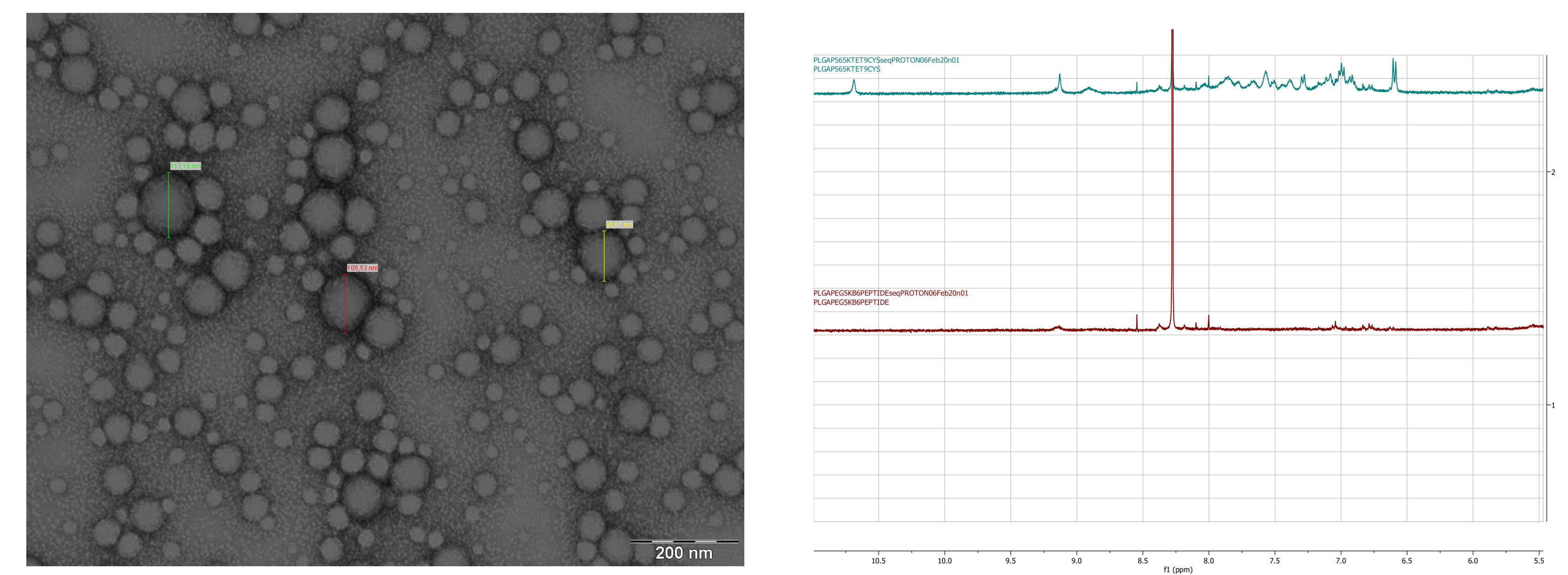

Fig. 4. TEM image of Lic-A NPs.

Fig. 6. ${ }^{1 H}$ NMR spectra of CPPs (TET-1-
polymer.

\section{CONCLUSIONS}

Morphometry of optimized Lic-A-PLGA NPs showed an average size of $159.3 \mathrm{~nm}$ with a PI less than 0.1 (characteristic of monodisperse systems), suitable to cross the BBB for brain delivery.

NPs developed have a spherical shape, with a smooth surface and without signs of aggregation phenomena, with a uniformly distributed dense pattern.

The results obtained by HPLC-ESI-MS corroborate a successful synthesis of the CPPs with a terminal thiol group.

\section{RESULTS}

Optimization of formulation by using a $2{ }^{4}$ factorial design, shows that average size of particles decreases for low concentrations of drug and surfactant, being in all cases less than $200 \mathrm{~nm}$ (Fig. 3). Table 1 shows the results of the physicochemical characteristics (average size, PI, ZP and EE) of the optimized of Lic-A NPs formulation. The TEM image (Fig. 4) obtained from the Lic-A NPs, reveals that they have a spherical shape, smooth surface with no signs of aggregation phenomena, being their average size similar to that obtained by PCS. The conjugation of the NPs with the CPP synthesized (Fig. 5) occurs by the covalent binding between the maleimide group of the derivatized polymer and the terminal thiol group of the CPP containing cysteine in its structure (SH-Cys) (Fig. 6).

Table 1. Effects of independent variables on Mean Size, PI, ZP and EE values of Lic-A NPs optimized formulation.
\begin{tabular}{|c|c|c|c|c|c|c|c|}
\hline $\begin{array}{c}\text { OPTIMIZED } \\
\text { FORMULATION }\end{array}$ & \multicolumn{3}{|c|}{ INDEPENDENT VARIABLES } & \multicolumn{4}{c|}{ DEPENDENT VARIABLES } \\
\cline { 2 - 8 } & Lic-A (mg/mL) & PLGA (mg/mL) & Tween $(\%)$ & Mean Size $(\mathrm{nm})$ & PI & ZP $(\mathrm{mV})$ & EE $(\%)$ \\
\hline Lic-A NPs & 1.0 & 8.0 & 0.4 & 159.3 & 0.095 & -35.5 & 56.3 \\
\hline
\end{tabular}

ACKNOWLEDGMENT: We acknowledge funding received from the Spanish Ministry of Economy, Industry and Competitiveness (MINECO) and the European Regional Development Fund (Grant RTI2018-094120-B-I00).

\section{REFERENCES}

(1) Abrego G., Alvarado H.L., Egea M.A., Gonzalez-Mira E., Calpena A.C., Garcia M.L. J. Pharm. Sci. 2014, 103, 3153-3164.

(2) Vasconcelos A., Vega E., Pérez Y., Gomara M.J., Garcla M.L., Haro I. Int. J. Nanomed. 2015, 10, 609-631. 